\title{
Optimal growth when tastes are inherited*
}

\author{
David de la Croix ${ }^{\dagger} \quad$ Philippe Michel $^{\ddagger}$ \\ First draft: March 1997 \\ This version: June 1997
}

\begin{abstract}
We address the issue of optimal growth when standard-of-living aspirations are transmitted from one generation to the next. We derive the condition for the optimal solution to be stable in the saddle-point sense and show that this optimal solution may display damped oscillations even when the planner does not discount the utility of future generations (golden rule case). The decentralization of the optimal solution aims at correcting the inter-generational externality by use of an investment subsidy and allows to avoid socially damaging rushes on consumption in expansion periods. It also allows to stabilize output in the case of competitive economies displaying endogenous fluctuations.
\end{abstract}

JEL Classification numbers: E32, O41.

Keywords: Externality, saddle-point stability, golden rule, oscillations, investment subsidy, stabilisation.

${ }^{*}$ We are grateful to Pascal Belan, Pierre Dehez, Alain Venditti and to the participants to the meeting "journées de générations imbriquées" in Marseille, March 97, for their helpful comments about an earlier draft.

${ }^{\dagger}$ National Fund for Scientific Research and IRES, Université catholique de Louvain, Place Montesquieu 3, B-1348 Louvain-la-Neuve, Belgium. E-mail: delacroix@ires.ucl.ac.be. The financial support of the PAC programme 93/98-162 of the Ministery of Scientific Research (French Speaking Community, Belgium) is gratefully acknowledged.

${ }^{\ddagger}$ IUF, Université de la Méditerrannée and GREQAM, Centre de la Vieille Charité, rue de la Charité 2, F-13002 Marseille, France. 


\section{Introduction}

Many empirical studies show that according to past actions consumers' tastes change over time (see e.g. Ferson and Constantinides, 1991). Frameworks with habit formation involve preferences that are "extended", according to Becker (1996)'s terminology, to account for the formation of personal and/or social capital. In the personal capital case, each agent's own history influences his current tastes and, hence, his decisions. Under the assumption of rational expectations, non-myopic agents forecast the change in their future preferences to compute the optimal decision (see the discussion in Pashardes (1986)). In the social capital case, the history of the society or of the social group to which the agent belongs influences his future tastes. This introduces a taste externality which is not taken into account in a decentralized world.

Both types of situations have been studied in the framemork of infinitely lived agents. On the one hand, Ryder and Heal (1973) analyse the personal capital case in a model à la Ramsey (1928) and show that the equilibrium can be characterized either by satiation or by the fulfilling of the modified golden rule. Various authors have used similar present value models with personal habits to understand problems linked to consumption behaviour. On the other hand, Abel (1990) and Campbell and Cochrane (1995) use the assumption of social capital to understand the so-called equity premium puzzle.

If we adopt the framework of finitely lived agents, or overlapping generations, a third type of habit capital emerges naturally: this is the case in which preferences should be extended to allow for familial capital. Under this assumption, children become habituated to a certain standard-of-living whilst still with their parents. Their past standard-of-living serves as a benchmark to evaluate the level of their own current consumption, once they have become adults and work. Becker (1992) notices that "the habits acquired as a child or young adult generally continue to influence behavior even when the environment changes radically. For instance, Indian adults who migrate to the United States often eat the same type of cuisine they had in India, and continue to wear the same type of clothing.(...) Childhood-acquired habits then continue, even though these would not have developed if the environment when growing up had been the same as the environment faced as an adult." Using a parametric example, de la Croix (1996) shows that this assumption can be responsible for oscillatory dynamics and endogenous cycles in a competitive economy. The intuition is that the aspirations of the new generation are so high at some point of an expansion that savings are depressed to maintain consumption standards, which induces the beginning of a contraction. The contraction stops when aspirations have reverted to lower levels.

In this paper, we adress the issue of optimal growth in the presence of inherited tastes. When parents are selfish, inherited tastes are responsible for an inter-generational externality and the inherited preferences are given to the children. "Children carry along into adulhood the baggage of experiences they had only a limited role in shaping. Therefore a rational person can meaningfully state that she does not like her preferences in the sense that she doesn't like the inherited baggage: the guilt, the sexual fears, the propensity to smoke or to drink heavely, and so forth." (Becker, 1992). This aspect may serve as a justification of making use of optimal solutions. After having derived the condition under which the competitive equilibrium with the taste externality displays fluctuations and/or explosive behaviour we look for the condition under which the optimal solution is stable in 
the saddle-point sense. One result will be that this optimal solution may display damped oscillations even when the planner does not discount the utility of future generations, i.e. in the golden rule case. Our result is surprising as the optimal solution is generally characterized by monotonous convergence in the one-sector overlapping generations model (see e.g. Deckert (1984)). This is in fact true as long as the utility function is separable both across generations and across periods of life. If the utility function is non-separable across periods of life, Michel and Venditti (1996) show that the eigenvalues of the system are still real but that convergence is not ensured any longer, so that endogenous cycles could appear. When the utility is non-separable across generations, but still separable across periods of life for simplicity, we show that (locally) converging oscillatory dynamics are highly likely.

We then study the decentralization of the optimal path, and hence, the stabilization of the competitive economy, by means of investment subsidies and lump-sum transfers. One result is that investment subsidies should always be positive, regardless of the sign of the effect of interest rate on savings. The optimal policy is then illustrated by the means of an example. After having computed the trajectories of both the optimal and the competitive economy, we want to compare the optimal policy for different values of the planner's discount rate. The question of the stabilization of the fluctuations (i.e. to what extent the fluctuations of the competitive economy are reduced by the optimal policy) is illustrated by our example.

The structure of the paper is as follows: the main assumptions of the model are presented in the first section. Some implications of the competitive solutions are drawn in section 2. The optimal solution is analysed in section 3, its decentralization and an example of optimal policy are presented in section 4. Section 5 discusses some further questions that arise in the framework of bequeathed tastes and concludes.

\section{The model}

At each date, the economy is populated by three generations, each living for three periods. The growth rate of the population is zero. The young generation inherits standard-ofliving aspirations $a_{t}$ from their parents, following

$$
a_{t}=c_{t-1}, \quad \forall t>0
$$

where $c_{t-1}$ is the consumption level of the parents. The adult generation draws utility from consuming the quantity $c_{t}$ given its own aspiration level $a_{t}$. When old, they will draw utility from consuming $d_{t+1}$ and die. The inter-temporal utility of the typical adult is:

$$
u\left(c_{t}, a_{t}\right)+v\left(d_{t+1}\right)
$$

We assume that bequeathed tastes determine a frame of reference against which consumption when adult is judged and that the depreciation rate of aspirations (i.e. forgetting) is high so that they no longer affect the evaluation of consumption when old. ${ }^{1}$

\footnotetext{
${ }^{1}$ This last assumption is supported by the empirical observation that reported satisfaction increases from the age of 30 onwards. Clark, Oswald and Warr (1994) concludes that "the rise in job satisfaction at the ages could come from reduced aspirations, due to a recognition that there are few alternative jobs available one a worker's career is established (...). Alternatively, aspirations themselves could remain the same but older workers might put less weight on such comparisons (...)."
} 
Moreover we assume $u_{c}, v_{d}>0 u_{a}<0, u_{c c}, u_{a a}, v_{d d}<0$ and $u_{c a}>0$. The assumption $u_{c a}>0$ amounts postulating that a rise in aspirations increases the marginal utility of (i.e. the desire for) consumption. We also assume that

$$
\lim _{d \rightarrow 0} v_{d}(d)=\infty
$$

and that $u$ is strictly concave:

$$
u_{c c} u_{a a}-u_{c a}^{2}<0
$$

At each date a single good is produced. This good can either be consumed by the middle-aged and old generation during the period or accumulated as capital for future production. Production occurs through a constant returns to scale technology. Output per capita $y_{t}$ is a function of capital intensity $k_{t}$ :

$$
y_{t}=f\left(k_{t}\right)
$$

in which $f($.$) is a neo-classical production function with f_{k}>0, f_{k k}<0$. Assuming that capital depreciates entirely after one period, the resource constraint of the economy is:

$$
y_{t}=c_{t}+d_{t}+k_{t+1}, \quad \forall t \geq 0
$$

At date 0 the economy is endowed with a given quantity of capital per capita $k_{0}$ and a level of aspirations $a_{0}$.

\section{The competitive economy}

We do not provide a complete analysis of the competitive economy with bequeathed tastes in this section, but show that a competitive economy may exhibit limit cycles based on a different mechanism than those already pointed in the literature. In particular, we show that the presence of cycles requires neither a negative effect of the interest rate on savings (as it is the case in Grandmont (1985)) nor low values of the elasticity of substitution in production (see Reichlin (1986)). Cycles result only from the fact that consumption is evaluated within a specific context, that is, the standard-of-living when young.

\subsection{The equilibrium}

The competitive behaviour of firms lead to the equalization of marginal productivities to marginal costs:

$$
\begin{aligned}
& R_{t}=f_{k}\left(k_{t}\right) \\
& w_{t}=f\left(k_{t}\right)-k_{t} f_{k}\left(k_{t}\right)
\end{aligned}
$$

where $R_{t}$ is the interest factor paid on loans and $w_{t}$ is the wage rate paid to workers.

The adult generation sells one unit of labour inelastically at any real wage $w_{t}$, consumes the quantity $c_{t}$ and saves $s_{t}$ for next period consumption by holding capital:

$$
c_{t}=w_{t}-s_{t}
$$


The old generation spends all its savings plus interest accrued and consumes $d_{t+1}$ :

$$
d_{t+1}=R_{t+1} s_{t}
$$

The maximisation programme of the individual is thus to choose $\left\{c_{t}, d_{t+1}\right\}$ in order to

$$
\max u\left(c_{t}, a_{t}\right)+v\left(d_{t+1}\right) \quad \text { subject to }(5),(6), \text { and } a_{t} \text { given. }
$$

This leads to the following first order condition:

$$
u_{c}\left(c_{t}, a_{t}\right)=R_{t+1} v_{d}\left(d_{t+1}\right)
$$

which allows us to define a saving function of the form:

$$
s_{t}=s\left(w_{t}, R_{t+1}, a_{t}\right)
$$

whose partial derivatives are

$$
s_{w}=\frac{u_{c c}}{u_{c c}+R_{t+1}^{2} v_{d d}}>0, \quad s_{r}=\frac{-\left(v_{d}+v_{d d} d_{t+1}\right)}{u_{c c}+R_{t+1}^{2} v_{d d}}, \quad s_{a}=\frac{u_{c a}}{u_{c c}+R_{t+1}^{2} v_{d d}}<0 .
$$

Since the utility function is concave and there is no wage income in the last period of life, savings increase with wage income. The effect of rising aspirations is to reduce savings, because it increases the desire for consumption when adult. The effect of the interest rate on savings is ambiguous and depends, as usual, on the value of the intertemporal elasticity of substitution, $v_{d d} d_{t+1} / v_{d}$.

The equilibrium condition on the capital market implies

$$
k_{t+1}=s_{t}
$$

Combining (1), (3), (4), (8) and (9), the competitive equilibrium is defined as a sequence $\left\{k_{t}, a_{t} ; t>0\right\}$ which satisfies:

$$
\left\{\begin{array}{l}
k_{t+1}=s\left(f\left(k_{t}\right)-k_{t} f_{k}\left(k_{t}\right), f_{k}\left(k_{t+1}\right), a_{t}\right) \\
a_{t+1}=f\left(k_{t}\right)-k_{t} f_{k}\left(k_{t}\right)-s\left(f\left(k_{t}\right)-k_{t} f_{k}\left(k_{t}\right), f_{k}\left(k_{t+1}\right), a_{t}\right) .
\end{array}\right.
$$

\subsection{The Naimarck-Sacker bifurcation}

Assuming that a positive steady state exists, we can show that a Naimark-Sacker ${ }^{2}$ bifur- $^{-}$ cation arises when the taste externality is strong enough. The Naimark-Sacker theorem is a powerful tool for the detection of limit cycles in discrete maps. Indeed a limit cycle appears either on the low or on the high side of the critical parameter value in a neighbourhood of the bifurcation point.

Let us consider a parameter $\gamma$ in the utility function: $u\left(c_{t}, a_{t}, \gamma\right)$. Then, assuming that $\gamma$ affects the marginal utilities, $\gamma$ appears in the savings function and the steady state can be written as $\{k(\gamma), a(\gamma)\}$. If at steady state we have

$$
s_{a}=\frac{1-s_{r} f_{k k}}{k f_{k k}},
$$

\footnotetext{
${ }^{2}$ This bifurcation is often called "Hopf bifurcation for maps".
} 


$$
s_{r}>\frac{1}{f_{k k}}
$$

and

$$
\frac{\left|s_{a}\right|}{\left(1-s_{r} f_{k k}\right)}<1+\left[1-\frac{s_{w} k\left|f_{k k}\right|}{1-s_{r}\left|f_{k k}\right|}\right],
$$

there is a Naimark-Sacker bifurcation in the parameter $\gamma$. The proof of this is given in Appendix A.

The conditions under which a Naimark-Sacker bifurcation arises are not too restrictive: the sufficient condition (12) assumes that the effect of the interest rate on savings is not "too" negative. In condition (13) (which is derived from the fact that the trace of the Jacobian of the linearized system should be lower than 2), the term in brackets is positive if the corresponding Diamond model is stable.

These conditions are satisfied in the simple example provided by de la Croix (1996): taking a Cobb-Douglas production function $y_{t}=k_{t}^{\alpha}$ and a utility of the form $\ln \left(c_{t}-\gamma a_{t}\right)+$ $\delta \ln d_{t+1}$, the Naimark-Sacker bifurcation occurs when

$$
\gamma=\frac{1+\alpha-\sqrt{(1+\alpha)^{2}-4 \alpha \delta /(\delta+1)}}{2 \alpha \delta /(\delta+1)}
$$

which is always between 0 and 1 .

It is theoretically possible to check whether the limit cycle that is implied by the Naimark-Sacker bifurcation theorem appears on the unstable side of the fixed point and is attracting or whether it encircles the fixed point on the stable side and is thus repelling. However, the computations involved are very lengthy (see Hale and Koçak, 1991) and the expressions depend on third derivatives of utility and production functions. As Farmer (1986) stresses, since economic theory does not place a priori restrictions on third derivatives, one can expect either repelling or attracting cycles to exist.

The spill-over from one generation to the next has two components: (a) the savings of the old generation finance the capital stock required for production as well as the wages of the adult generation; this process transforming income/savings of the old into income for the adult displays decreasing returns; (b) past consumption levels of the parents generate standard-of-living aspirations for the young generation, inducing them to spend more on consumption; this process displays constant returns. The interpretation of the existence of cycles in the competitive economy is the following: due to the decreasing returns in the production process, the bequest in terms of higher wages will not sufficient to cover the bequest in terms of higher aspirations at some point in time. This leads to a drop in savings to maintain the standard-of-living and thus induces a recession. When the consecutive impoverishment is strong enough, aspirations have reverted to lower levels, allowing a rise in savings and the start of an expansion period. Depending on the relative strength of the two effects, this process can either converge, explode or lead to everlasting cycles.

\section{The optimal solution}

Let us now turn our attention to the optimal solution and consider a central planner who chooses the allocation of output in order to maximize the present discounted value of 
current and future generations. Assuming that the central planner's discount factor is $\beta$, the social welfare function takes the following form:

$$
W=v\left(d_{0}\right)+\sum_{t=0}^{\infty} \beta^{t+1}\left[u\left(c_{t}, a_{t}\right)+v\left(d_{t+1}\right)\right]
$$

The planner chooses $\left\{c_{t}, d_{t} ; t \geq 0\right\}$ and $\left\{k_{t}, a_{t} ; t>0\right\}$ so as to maximize

$$
\sum_{t=0}^{\infty} \beta^{t+1}\left[u\left(c_{t}, a_{t}\right)+\frac{1}{\beta} v\left(d_{t}\right)\right] \text { s.t. (1), (2), } a_{0} \text { and } k_{0} \text { given. }
$$

The first order conditions for a maximum are:

$$
\begin{gathered}
u_{c}\left(c_{t}, a_{t}\right)+\beta u_{a}\left(c_{t+1}, a_{t+1}\right)=\frac{1}{\beta} v_{d}\left(d_{t}\right) \\
\frac{1}{\beta} v_{d}\left(d_{t}\right)=v_{d}\left(d_{t+1}\right) f_{k}\left(k_{t+1}\right)
\end{gathered}
$$

The first equation is a condition for the optimal allocation of consumption between the adult and the old generation which are alive at the same time. The marginal utility of consumption of adults corrected to internalize the taste externality is equalized to the marginal utility of consumption of the old. Note that, due to the presence of the taste externality and contrary to the standard Diamond (1965) model, this planner's first-order condition does not respect the first-order condition the individual chooses for himself in a market economy (equation (7)). The second equation is the usual condition describing the optimal intertemporal allocation.

The steady state $\{c, a, k, d\}$ of this optimal economy is defined by:

$$
\begin{aligned}
f_{k}(k) & =\beta^{-1} \\
u_{c}(c, a) & =\beta^{-1} v_{d}(d)-\beta u_{a}(c, a) \\
a & =c \\
f(k) & =d+c+k
\end{aligned}
$$

Equation (16) is the modified golden rule. Hence, the introduction of bequeathed tastes does not modify the optimal steady state stock of capital which remains fixed at the modified golden rule level. Equation (17) shows that the marginal utility of adults should be larger in the economy with inherited tastes than in the Diamond (1965) economy, this implies that they should consume less.

\section{Proposition 1. [Existence of the steady state(s)]}

A sufficient condition for the existence of a steady state satisfying the system (16)-(19) is

$$
\lim _{c \rightarrow 0}\left[u_{c}(c, c)+\beta u_{a}(c, c)-\frac{1}{\beta} v_{d}\left(b^{\star}-c\right)\right]>0
$$

in which $b^{\star}=c^{\star}+d^{\star}=f\left(k^{\star}\right)-k^{\star}$ with $k^{\star}$ statisfying $\beta f_{k}\left(k^{\star}\right)=1$. 
Proof: let us define

$$
\phi(c)=u_{c}(c, c)+\beta u_{a}(c, c)-\beta^{-1} v_{d}\left(b^{\star}-c\right) .
$$

Steady state consumption, $c^{\star}$, is the solution of $\phi\left(c^{\star}\right)=0$. Notice that

$$
\lim _{d \rightarrow 0} v_{d}(d)=\infty \quad \Longrightarrow \quad \lim _{c \rightarrow b^{\star}} \phi(c)=-\infty
$$

Since $\phi(c)$ is continuous, there exists a $c^{\star}$ such that $\phi\left(c^{\star}\right)=0$ if and only if there exists at least one $c$ such that $\phi(c) \geq 0$. This is guarantueed for instance if $\lim _{c \rightarrow 0} \phi(c)>0$.

Equation (20) states that the negative effect of habits does not offset the gain in welfare linked to a rise in consumption, when $c=0$. In particular, the existence of the steady state is guaranteed as long as the world is not Veblenian: Veblen believed that the welfare of a typical person primarily depends on his relative income position. In that case, the value of a social capital causing envy exactly offsets the value of own consumption. A rise in all incomes in a community by the same percentage would not improve anyone's welfare in Veblen's world (see Becker (1974), Veblen (1934)).

Condition (20) is satisfied e.g. when $\lim _{c \rightarrow 0} u_{c}(c, c)+u_{a}(c, c)=+\infty$.

\section{Proposition 2. [Unicity of the steady state]}

A sufficient condition for unicity of the steady state satisfying the system (16)-(19) is

$$
u_{c c}+(1+\beta) u_{c a}+\beta u_{a a} \leq 0
$$

Proof: Using the same definition of $\phi$ as in Proposition 1, unicity is obtained if $\phi_{c}(c)<0, \forall c$ i.e. if

$$
u_{c c}+(1+\beta) u_{c a}+\beta u_{a a}+\beta^{-1} v_{d d}<0
$$

This is always guaranteed for instance if $u_{c c}+(1+\beta) u_{c a}+\beta u_{a a} \leq 0 . \square$

Notice that given the concavity of $u(c, a)$, for $\beta=1$ condition (21) is always satisfied. In the other cases, (21) imposes a restriction on $u_{c a}$ conditionally on $\beta, u_{c c}$ and $u_{a a}$ :

$$
u_{c a} \leq \frac{\left|u_{c c}\right|+\beta\left|U_{a a}\right|}{1+\beta}
$$

This condition is satisfied for every $\beta$ 's as long as $u_{c a} \leq\left|u_{c c}\right|$ and $u_{c a} \leq\left|u_{a a}\right|$. If not, there is a restriction on $\beta$.

Turning our attention to the dynamics of the optimal economy, we see that it can be described by the following system of four first-order non-linear difference equations:

$$
\left\{\begin{aligned}
\beta u_{a}\left(c_{t+1}, c_{t}\right) & =\frac{1}{\beta} v_{d}\left(d_{t}\right)-u_{c}\left(c_{t}, a_{t}\right) \\
v_{d}\left(d_{t+1}\right) f_{k}\left(k_{t+1}\right) & =\frac{1}{\beta} v_{d}\left(d_{t}\right) \\
a_{t+1} & =c_{t} \\
k_{t+1} & =f\left(k_{t}\right)-d_{t}-c_{t}
\end{aligned}\right.
$$


in which $a_{t}$ and $k_{t}$ are predetermined and $c_{t+1}$ and $d_{t+1}$ are anticipated variables. For the equilibrium defined by (22) to be unique and (locally) converging to the steady state, since there are two anticipated variables in the system two (and only two) eigenvalues of its linearization should have a modulus larger than one.

\section{Proposition 3. [Stability of the steady state]}

Under condition (21), the stationary state is a saddle-point. Let us define at steady state

$$
\Delta \equiv\left[u_{c c}+\beta u_{a a}+u_{c a}\left(1+\beta+\frac{\beta^{2} v_{d}}{v_{d d}} f_{k k}\right)\right]^{2}+4 \beta u_{c a} v_{d} f_{k k}
$$

If $\Delta>0$, the four eigenvalues are real and the local dynamics are monotonous. If $\Delta<0$, the eigenvalues are complex and the local dynamics display damped oscillations.

Proof: see Appendix B $\square$

The condition $\Delta>0$ is always satisfied if e.g. $u_{c a}=0$, which corresponds to the case in which inherited tastes do not affect the marginal utility of consumption when adult. We thus retrieve the standard result of optimal growth. More surprisingly, the condition $\Delta>0$ is satisfied if $v_{d d}$ or $f_{k k}$ is close enough to zero, i.e. if $v($.$) and f($.$) are not too$ concave. Notice also that, even when $\beta=1$, i.e. the planner does not discount the future at all, the condition $\Delta>0$ is not necessarily satisfied. This implies that the optimal dynamics around the golden rule can be characterised by oscillations.

\section{Decentralizing the first best solution}

\subsection{Optimal taxation}

The decentralization of the first best solution aims at two objectives: (a) to adjust the adults' consumption of in order to internalize the externality; (b) to adjust savings to match the level of the modified golden rule.

This decentralization can be achieved by means of an investment subsidy $i_{t}>1$ and by using lump sum transfers to the adults, $g_{t}$, and to the old, $p_{t}$. An alternative would be to tax the consumption of the adult generation and that of the old differently, but such a discrimination does not seem fortunate.

The maximisation programme of the individual becomes

$$
\max u\left(c_{t}, a_{t}\right)+v\left(d_{t+1}\right)
$$

$$
\begin{aligned}
\text { subject to } \quad \begin{aligned}
c_{t} & =w_{t}+g_{t}-s_{t} \\
d_{t+1} & =R_{t+1} i_{t+1} s_{t}+p_{t+1} \\
a_{t} & \text { given }
\end{aligned}
\end{aligned}
$$


This leads to the following first order condition:

$$
u_{c}\left(c_{t}, a_{t}\right)=R_{t+1} i_{t+1} v_{d}\left(d_{t+1}\right)
$$

\section{Proposition 4. [Positive investment subsidy]}

To decentralize the first best solution, investment subsidies should always be positive regardless of the sign of the effect of interest rates on savings.

Proof: Comparing (23) to (14) and (15), it appears that the investment subsidy should satisfy:

$$
i_{t+1}=\left(1+\beta u_{a}\left(c_{t+1}^{\star}, a_{t+1}^{\star}\right) / u_{c}\left(c_{t}^{\star}, a_{t}^{\star}\right)\right)^{-1}
$$

in which starred variables denote the optimal levels. Using (14), it is clear that this expression is always positive, whatever the effect of interest rates on savings.

Comparing the competitive equilibrium to the optimal solution described in (22), it appears that the two remaining instruments should follow:

$$
\begin{aligned}
& g_{t}=k_{t+1}^{\star}+c_{t}^{\star}-w_{t}^{\star} \\
& p_{t}=-g_{t}-\left(i_{t}-1\right) R_{t}^{\star} k_{t}^{\star}
\end{aligned}
$$

Equation (25) ensures that savings and, hence, the capital stock are set at the level of the modified golden rule. Equation (26) is the planner's budget constraint.

\subsection{Example}

It is interesting to illustrate these results by an example. We shall compute the optimal trajectories of the optimal economy for two different planner's discount rates and compare them to the trajectory of the competitive economy. We also provide the dynamic path of optimal taxes and subsidies.

The intertemporal utility of the consumer is given by:

$$
\ln \left(c_{t}-\gamma a_{t}\right)+\delta \ln \left(d_{t+1}\right)
$$

The production function is given by $y_{t}=\tau k_{t}^{\alpha}$. Taking $\delta=.43, \alpha=.25, \tau=10$ and $\gamma=.65$, the steady state output of the optimal economy is 13.527 when $\beta=.99$ and 10.772 when $\beta=.5$. Steady state output of the economy with perfect competition is 9.927. The eigenvalues of the linearized systems are presented in table 1 . We see that the unique optimal trajectory locally converging to the steady state displays damped oscillations, even when $\beta=.99$. This illustrates the fact that a high planner's discount factor does not prevent the optimal economy from displaying fluctuations.

Starting with a negligible initial capital stock and low aspirations, the equilibrium of the optimal economy and of the competitive economy are presented in figures 1 and 2 for two different values of $\beta$. The simulations are performed using the methodology proposed by Boucekkine (1995) for saddle-point trajectories. The infinite horizon problem 
Figure 1: The optimal $(\beta=.99)$ and the competitive economies
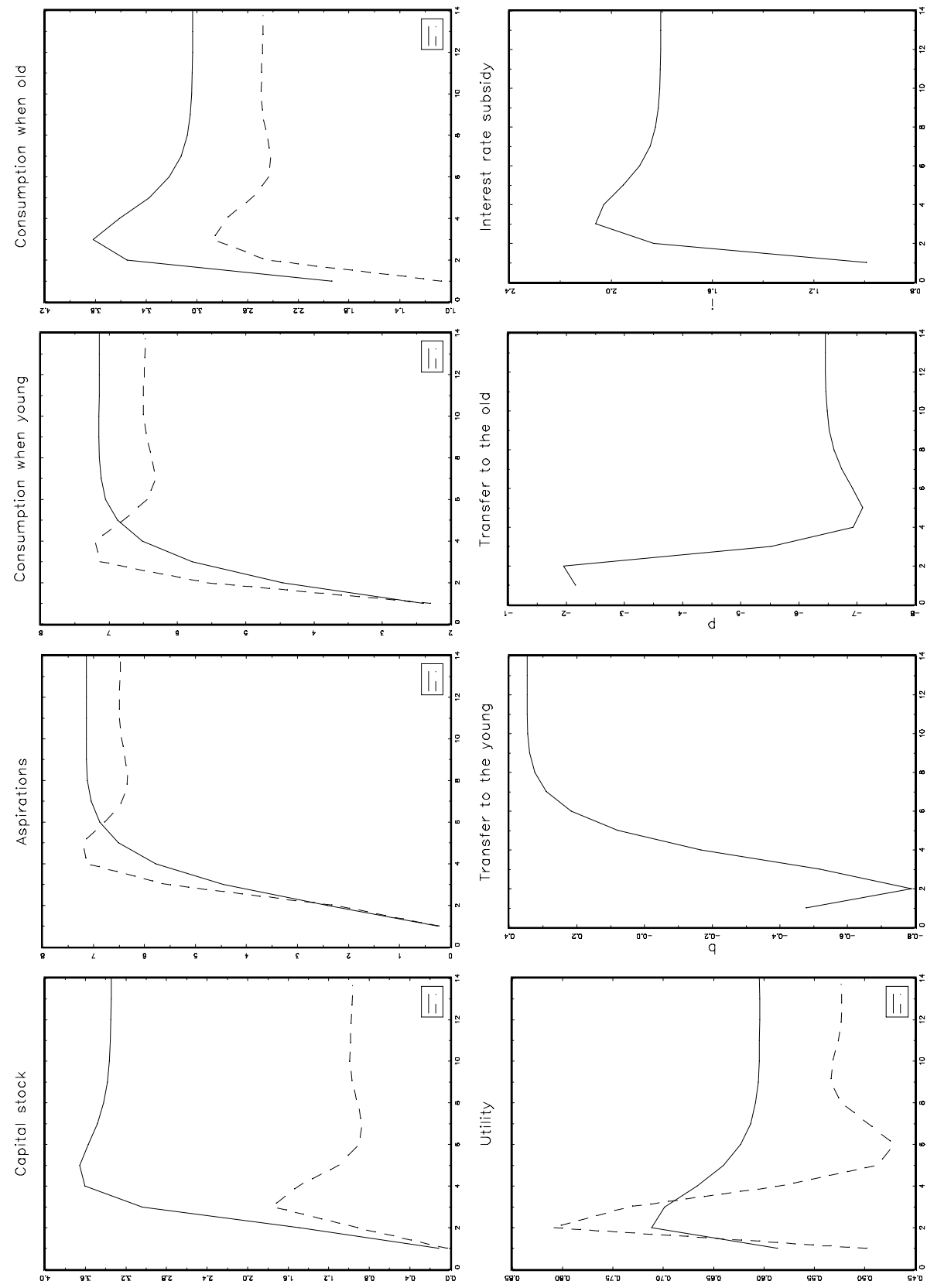
Figure 2: The optimal $(\beta=.5)$ and the competitive economies
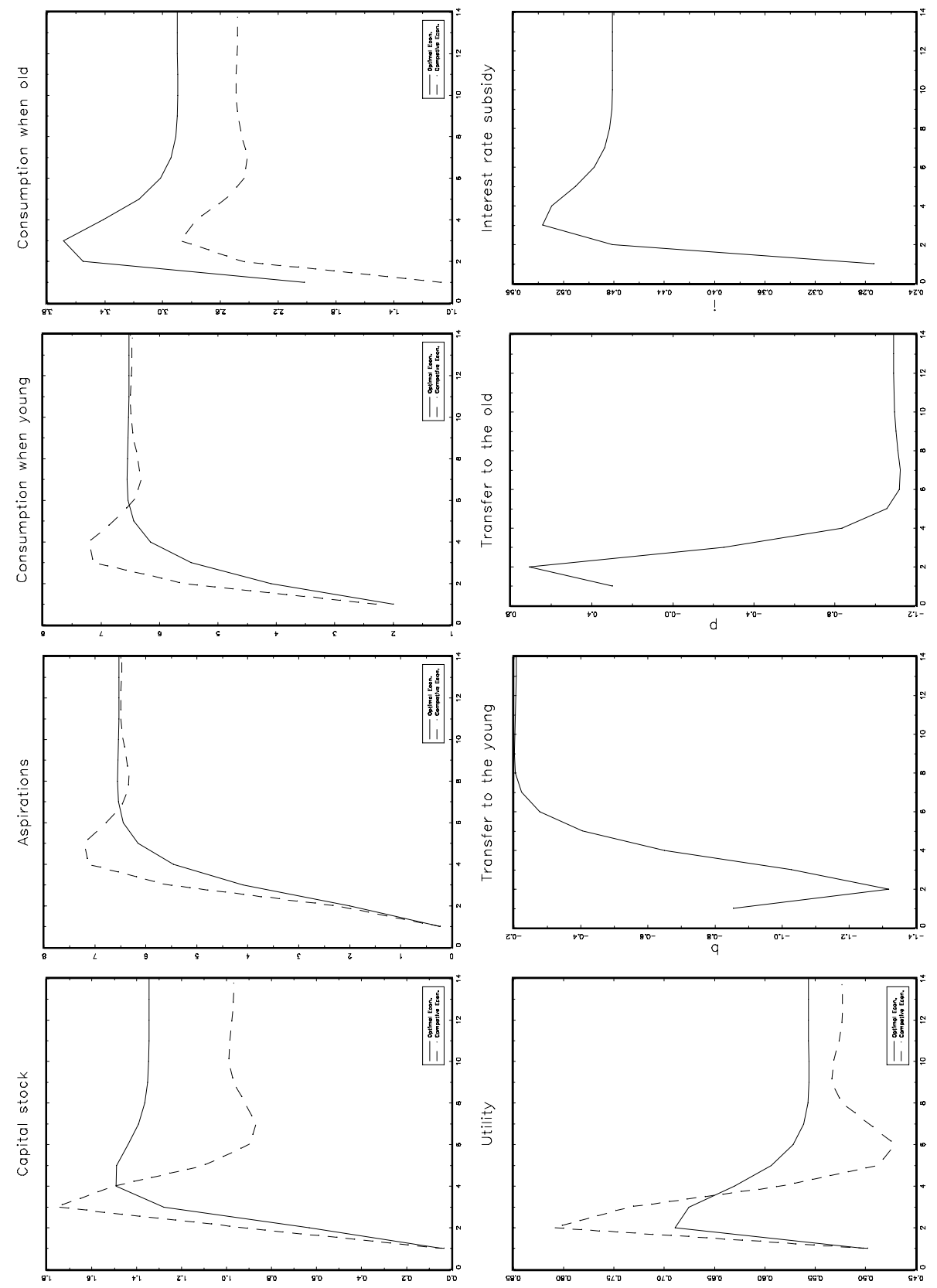
Table 1: Eigenvalues: examples

\begin{tabular}{lll} 
optimal economy $(\beta=.99)$ & $0.4587 \pm 0.1503 i$ & $1.9883 \pm 0.6515 i$ \\
optimal economy $(\beta=.5)$ & $0.4373 \pm 0.2073 i$ & $3.7339 \pm 1.7710 i$ \\
competitive economy & $0.3850 \pm 0.4749 i$ & \\
\hline
\end{tabular}

is approximated by a horizon of 100 periods. Increasing the simulation horizon further does not modify the results. Only the first 14 periods are displayed since the steady state is almost attained after 14 periods.

Considering first the competitive economy (dashed lines, identical in the two figures), aspirations at first increase rapidly. After three periods of growth the adults start saving less in order to maintain a sustained increase in consumption. The capital stock starts declining at $t=3$ as aspirations increase faster than wages, the latter being subject to decreasing marginal returns. After period 3, wages and interest revenues start to decrease in line with the capital stock, which depresses consumption of both adult and old agents. When aspirations have reverted to sufficiently low levels (at $t=6$ ) the capital stock starts increasing again and the economy converges to its steady state with damped oscillations.

It is clear from figure 1 that, at steady state, capital, aspirations and consumption are higher in the optimal economy of the quasi golden rule type $(\beta=.99)$ than in the competitive economy. To obtain this allocation, the optimal policy consists of subsidizing savings in order to initially lower the rise in consumption and hence avoid the rush on it by the adult generations. These subsidies are financed by lump-sum taxes bearing essentially on the old agents, implying that what is given by one hand in a proportional way is taken by the other in a lump-sum fashion. Notice finally that, in the growth process, the utility of the generations born in $t+2$ and $t+3$ is higher in the competitive economy, but is at the expense of all future generations.

The comparison of figure 2 with figure 1 shows that investment subsidies are lower when $\beta=.5$ than in the case $\beta=.99$. Moreover, in the quasi golden rule case, the transferts from the olds to the adults are higher. Indeed, these transferts are useful to increase the capital stock which benefits to all future generations.

\section{Conclusion}

We have studied a model in which utility is non-separable across generations and familial capital in terms of tastes is transmitted from one generation to another. This framemork introduces an externality that is not taken into account by non-altruistic parents and may be responsible for endogenous fluctuations in competitive economies.

We have adressed the issue of optimal growth in this case. Having studied the dynamic system corresponding to the optimal growth problem, we show that the solution is always stable in the saddle-point sense provided that the world is non-Veblenian, i.e. that the 
welfare of a typical agent does not only depend on its relative position. We also show that this optimal solution may display oscillations even when the planner does not discount the utility of future generations, that is, in the golden rule case.

The decentralization of the optimal solution aims at correcting the inter-generational externality and can be achieved with three instruments. A sufficient policy is indeed to subsidize savings in order to avoid socially unsustainable rushes on consumption by the adult generations. The decentralization also allows to stabilize the economy, therefore avoiding endogenous fluctuations.

An interesting extension would be to consider altruistic agents. In that case, even if the non-zero bequest condition is binding, altruism has an influence on the economy: parents could restrain themselves to make their children less demanding and hence increase their welfare.

Finally, keeping in mind that the presence of positive externalities is at the root of endogenous growth models, the introduction of bequeathed tastes adds a negative externality to the model. Another interesting extension could then be to investigate the conditions under which one type of externality dominates the other, making unbounded growth optimal or not.

\section{Appendix A}

Assuming that at least one non-zero steady state exists, $\{k(\gamma), a(\gamma)\}$, the linearization of the dynamic system gives:

$$
\left[\begin{array}{c}
\mathrm{d} k_{t+1} \\
\mathrm{~d} a_{t+1}
\end{array}\right]=\frac{1}{1-s_{r} f_{k k}}\left[\begin{array}{cc}
-s_{w} k f_{k k} & s_{a} \\
\left(s_{w}-1+s_{r} f_{k k}\right) k f_{k k} & -s_{a}
\end{array}\right]\left[\begin{array}{c}
\mathrm{d} k_{t} \\
\mathrm{~d} a_{t}
\end{array}\right]
$$

The trace and the determinant of the jacobian matrix are:

$$
T=\frac{-s_{a}-s_{w} k f_{k k}}{1-s_{r} f_{k k}}, D=\frac{s_{a} k f_{k k}}{1-s_{r} f_{k k}}
$$

The condition

$$
s_{r}>\frac{1}{f_{k k}}
$$

is sufficient for the trace and the determinant to be positive. The condition

$$
\frac{\left|s_{a}\right|}{1-s_{r} f_{k k}}<1+\left[1-\frac{s_{w} k\left|f_{k k}\right|}{1-s_{r}\left|f_{k k}\right|}\right]
$$

is sufficient for the trace to be smaller than 2. In that case, following Hale and Koçak (1991), if

$$
s_{a}=\frac{1-s_{r} f_{k k}}{k f_{k k}}
$$

it can be checked that (i) the determinant is equal to 1, (ii) the discriminant of the Jacobian matrix is negative (because the trace is $\epsilon]-2,2$ [, (iii) the two non-real eigenvalues cross the unit circle at a non-zero speed when the parameter $\gamma$ changes and that (iv) none of them may be one of the first four roots of unity (excluding cases of weak resonance). These four conditions are sufficient to identify a Naimark-Sacker bifurcation. 


\section{Appendix B}

Linearizing (22) around the steady state leads to:

$$
\left[\begin{array}{c}
\mathrm{d} a_{t+1} \\
\mathrm{~d} c_{t+1} \\
\mathrm{~d} k_{t+1} \\
\mathrm{~d} d_{t+1}
\end{array}\right]=\left[\begin{array}{cccc}
0 & 1 & 0 & 0 \\
-1 / \beta & A & 0 & -C \\
0 & -1 & 1 / \beta & -1 \\
0 & B & -B / \beta & 1+B
\end{array}\right]\left[\begin{array}{c}
\mathrm{d} a_{t} \\
\mathrm{~d} c_{t} \\
\mathrm{~d} k_{t} \\
\mathrm{~d} d_{t}
\end{array}\right]
$$

in which

$$
A=-\frac{u_{c c}+\beta u_{a a}}{\beta u_{c a}}, \quad B=\frac{\beta v_{d} f_{k k}}{v_{d d}}, \quad C=\frac{-v_{d d}}{\beta^{2} u_{c a}} .
$$

The determinant of the Jacobian matrix is $1 / \beta^{2}$ and the trace is $T=1+A+B+1 / \beta$. Notice that $T>0$. The characteristic polynomial is:

$$
x^{4}-T x^{3}+Z x^{2}-T \beta^{-1} x+\beta^{-2}
$$

with $Z=1 / \beta+A / \beta+A+A B-C B$. Following Levhari and Liviatan (1972), it can be factorized into

$$
\left(x-x_{1}\right)\left(x-\frac{1}{\beta x_{1}}\right)\left(x-x_{2}\right)\left(x-\frac{1}{\beta x_{2}}\right)
$$

where $x_{1}, \frac{1}{\beta x_{1}}, x_{2}$ and $\frac{1}{\beta x_{2}}$ are the eigenvalues. The above expression can be rewritten as:

$$
\left(x^{2}-\sigma_{1} x+\frac{1}{\beta}\right)\left(x^{2}-\sigma_{2} x+\frac{1}{\beta}\right)
$$

These eigenvalues are solution of

$$
x_{i}^{2}-\sigma_{i} x_{i}+\frac{1}{\beta}=0, \quad i=1,2
$$

$\sigma_{1}$ and $\sigma_{2}$ being solution of

$$
\sigma^{2}-T \sigma+P=0
$$

with $P=Z-2 / \beta$.

Let us also define

$$
D \equiv A-(1+1 / \beta)=-\frac{1}{\beta u_{c a}}\left(u_{c c}+\beta u_{a a}+(1+\beta) u_{c a}\right)
$$

The hypothesis (21) can be written $D \geq 0$.

The discriminant of equation (28) is:

$$
\Delta=T^{2}-4 P=T^{2}-4 A T+4 A^{2}-4 B C=(T-2 A)^{2}-4 B C=(B-D)^{2}-4 B C
$$

- If $\Delta \geq 0$, then $\sigma_{1}=(T-\sqrt{\Delta}) / 2 \leq \sigma_{2}=(T+\sqrt{\Delta}) / 2$ are real.

We have also that

$$
\sigma_{1}-(1+1 / \beta)=(T-\sqrt{\Delta}-2(1+1 / \beta)) / 2=(B+D-\sqrt{\Delta}) / 2 .
$$

Under hypothesis $(21), D \geq 0,(B+D)^{2}>(B-D)^{2}-4 B C=\Delta$ and $\sigma_{1}-(1+1 / \beta)>0$. A fortiori, $\sigma_{2}-(1+1 / \beta)>0$. The polynomial (5) is negative at $x_{i}=1$ and thus admits one root $x_{i}$ such that $0<x_{i}<1$. We thus have two real stable roots, $x_{1}$ and $x_{2}$, (and hence two real unstable roots $\frac{1}{\beta x_{1}}$ and $\frac{1}{\beta x_{2}}$ ) ensuring a monotonous (local) convergence. 
- If $\Delta<0, \sigma_{1}$ and $\sigma_{2}$ are complex conjugates with modulus $\left|\sigma_{i}\right|^{2}=P . \sigma_{1}=x_{1}+\frac{1}{\beta x_{1}}$ is non-real. We also have that

$$
P=\left(x_{1}+\frac{1}{\beta x_{1}}\right)\left(\overline{x_{1}}+\frac{1}{\beta \overline{x_{1}}}\right)=h+\frac{1}{\beta^{2} h}+\frac{2}{\beta}
$$

where $h=\left|x_{1}\right|^{2}$ is the solution of

$$
h^{2}+\left(\frac{2}{\beta}-P\right) h+\frac{1}{\beta^{2}}=0
$$

There exist a solution $h, 0<h<1$ if and only if

$$
1+\frac{2}{\beta}-P+\frac{1}{\beta^{2}}<0 \Longleftrightarrow P>\left(1+\frac{1}{\beta}\right)^{2}
$$

We have that

$$
P-\left(1+\frac{1}{\beta}\right)^{2}=A\left(1+\frac{1}{\beta}+B\right)+B C-\left(1+\frac{1}{\beta}\right)^{2}=\left(1+\frac{1}{\beta}\right) D+B A+B C
$$

which is positive under assumption (21). We thus have a saddle-point with fluctuations.

\section{References}

Abel, A. (1990) "Asset prices under habit formation and catching up with the Joneses". American Economic Review Papers and Proceedings, 80:38-42.

Becker, G. (1974) "A theory of social interactions". Journal of Political Economy, 82:1063-1091.

Becker, G. (1992) "Habits, addictions and traditions". Kyklos, 45:327-345.

Becker, G. (1996) Accouting for tastes. Harvard University Press.

Boucekkine, R. (1995) "An alternative methodology for solving non-linear forwardlooking models". Journal of Economic Dynamics and Control, 19:711-734.

Campbell, J. and J. Cochrane (1995) "By force of habits: a consumption-based explanation of aggregate stock market behavior". Working Paper, NBER.

Clark, A., A. Oswald and P. Warr (1994) "Is job-satisfaction U-shaped in age?" Working Paper, CEPREMAP.

de la Croix, D. (1996) "The dynamics of bequeathed tastes". Economics Letters, 51:8996.

Deckert, W. (1984) "Does optimal growth preclude chaos ? a theorem on monotonicity". Zeitschrift fur National Okonomie, 44:57-61.

Diamond, P. (1965) "National debt in a neoclassical growth model". American Economic Review, 55:1126-1150.

Farmer, R. (1986) "Deficits and cycles". Journal of Economic Theory, 40:77-88. 
Ferson, W. and G. Constantinides (1991) "Habit persistence and durability in aggregate consumption". Journal of Financial Economics, 29:199-240.

Grandmont, J.-M. (1985) "On endogenous competitive business cycles". Econometrica, 22:995-1037.

Hale, J. and H. Koçak (1991) Dynamics and bifurcation. Springer Verlag.

Levhari, D. and N. Liviatan (1972) "On stability in the saddle-point sense". Journal of Economic Theory, 4:88-93.

Michel, P. and A. Venditti (1996) "Optimal growth and cycles in overlapping generations models". Economic Theory, forthcoming.

Pashardes, P. (1986) "Myopic and forward looking bahaviour in a dynamic demand system". International Economic Review, 27:387-397.

Ramsey, F. (1928) "A mathematical theory of savings". Economic Journal, 38:543-559.

Reichlin, P. (1986) "Equilibrium cycles in an overlapping generations economy with production". Journal of Economic Theory, 40:89-102.

Ryder, H. and G. Heal (1973) "Optimal growth with inter-temporally dependent preferences". Review of Economic Studies, 40:1-31.

Veblen, T. (1934) The theory of the leisure class. Modern Library. 